\title{
A $\beta$-Camera Integrated with a Microfluidic Chip for Radioassays Based on Real-Time Imaging of Glycolysis in Small Cell Populations
}

\author{
Nam T. Vu ${ }^{1,2}$, Zeta T.F. Yu ${ }^{1,2}$, Begonya Comin-Anduix ${ }^{3,4}$, Jonas N. Søndergaard ${ }^{5}$, Robert W. Silverman ${ }^{1,2}$, \\ Canny Y.N. Chang ${ }^{1}$, Antoni Ribas ${ }^{3-5}$, Hsian-Rong Tseng ${ }^{1,2}$, and Arion F. Chatziioannou ${ }^{1,2}$ \\ ${ }^{1}$ Crump Institute for Molecular Imaging, University of California, Los Angeles, California; ${ }^{2}$ Department of Molecular and Medical \\ Pharmacology, University of California, Los Angeles, California; ${ }^{3}$ Division of Surgical Oncology, Department of Surgery, University \\ of California, Los Angeles, California; ${ }^{4}$ Jonsson Comprehensive Cancer Center, University of California, Los Angeles, California; \\ and ${ }^{5}$ Division of Hematology/Oncology, Department of Medicine, University of California, Los Angeles, California
}

An integrated $\beta$-camera and microfluidic chip was developed that is capable of quantitative imaging of glycolysis radioassays using ${ }^{18} \mathrm{~F}-\mathrm{FDG}$ in small cell populations down to a single cell. This paper demonstrates that the integrated system enables digital control and quantitative measurements of glycolysis in $B-R a f^{V 600 E}$-mutated melanoma cell lines in response to specific B-Raf inhibition. Methods: The $\beta$-camera uses a positionsensitive avalanche photodiode to detect charged particleemitting probes within a microfluidic chip. The integrated $\beta$-camera and microfluidic chip system was calibrated, and the linearity was measured using 4 different melanoma cell lines (M257, M202, M233, and M229). Microfluidic radioassays were performed with cell populations ranging from hundreds of cells down to a single cell. The M229 cell line has a homozygous BRaf $^{\mathrm{V} 600 E}$ mutation and is highly sensitive to a B-Raf inhibitor, PLX4032. A microfluidic radioassay was performed over the course of 3 days to assess the cytotoxicity of PLX4032 on cellular ${ }^{18}$ F-FDG uptake. Results: The $\beta$-camera is capable of imaging radioactive uptake of ${ }^{18} \mathrm{~F}-\mathrm{FDG}$ in microfluidic chips. ${ }^{18} \mathrm{~F}-\mathrm{FDG}$ uptake for a single cell was measured using a radioactivity concentration of $37 \mathrm{MBq} / \mathrm{mL}$ during the radiotracer incubation period. For in vitro cytotoxicity monitoring, the $\beta$-camera showed that exposure to $1 \mu \mathrm{M}$ PLX4032 for 3 days decreased the ${ }^{18} \mathrm{~F}$-FDG uptake per cell in highly sensitive M229 cells, compared with vehicle controls. Conclusion: The integrated $\beta$-camera and microfluidic chip can provide digital control of live cell cultures and allow in vitro quantitative radioassays for multiple samples simultaneously.

Key Words: instrumentation; microfluidics; radioassay; ${ }^{18} \mathrm{~F}-$ FDG; PET

J Nucl Med 2011; 52:815-821

DOI: 10.2967/jnumed.110.078725

\footnotetext{
Received May 3, 2010; revision accepted Jan. 7, 2011.

For correspondence or reprints contact: Arion F. Chatziioannou, Department of Molecular and Medical Pharmacology, Crump Institute for Molecular Imaging, UCLA, P.O. Box 951770, 4345 CNSI, Los Angeles, CA 90095-1770. E-mail: archatziioann@mednet.ucla.edu

Guest Editor: David Townsend, University of Tennessee Medical Center COPYRIGHT @ 2011 by the Society of Nuclear Medicine, Inc.
}

$\mathbf{M}$ olecular imaging tools such as PET can provide in vivo measurements of biochemical processes in tissue to reveal the status and monitor the therapeutic response of disease, for example, cancer (1). However, complicating factors such as tissue microenvironment (2), body clearance, cell heterogeneity, and technologic limitations in sensitivity and spatial resolution prohibit accurate measurements of biochemical processes in subpopulations and single cells. Alternatively, in vitro radioassays can provide a better connection to more specific cellular functions, such as glycolysis (3), which can be correlated with physiologic states of therapeutic responses. Changes in cellular metabolic state-for example, the many types of cancer cells that exhibit increased glycolysis rates, compared with normal cells_-can be linked to several diseases $(4,5)$.

Current technologies for in vitro radioassays can provide high sensitivity for detection of radiotracers; however, they rely on macroscopic systems, thereby limiting the level of control for small populations or single-cell cultures (6). The use of microfluidic technologies can provide a platform for integrated, digital control of small volumes of reagents and samples suitable for bioassays of small cell populations. Recent microfluidic bioassays have demonstrated the ability to measure concentrations of multiplesignal proteins in single cells among heterogeneous populations (7), low-copy-number proteins in single cells (8), and intracellular calcium ion concentrations in single cells (9). Although many techniques are available for measuring biochemical functions in microfluidic systems, the use of radiometric methods can provide high sensitivity for small amounts of radiotracers. Furthermore, radiolabeled probes that adhere to the composition and structure of the target molecule can be readily translated to clinical applications. Thus, a microfluidic radioassay platform for measuring cellular ${ }^{18} \mathrm{~F}-\mathrm{FDG}$ uptake can complement conventional clinical techniques such as ${ }^{18} \mathrm{~F}-\mathrm{FDG}$ PET and enable monitoring of glycolysis in response to novel clinical therapies. 
Oncogenic mutations in cancer profoundly affect cellular metabolism with the activation of the Warburg effect (10), whereas oncogene inhibition with novel therapies can alter the metabolic signatures. This effect could be particularly important for the monitoring of antitumor effects of novel treatments in cancer histologies with high ${ }^{18} \mathrm{~F}-\mathrm{FDG}$ uptake, as has been demonstrated with mutations in the mitogenactivated protein kinase pathway (11). The $\mathrm{B}-\mathrm{Raf}^{\mathrm{V} 600 \mathrm{E}}$ oncogenic mutation is present in $60 \%-70 \%$ of melanomas and leads to uncontrolled cell growth $(12,13)$ and increased cellular glucose metabolism (11). There are several B-Raf inhibitors in clinical development with evidence of inducing response rates in over $70 \%$ of patients with melanoma harboring the B-Raf ${ }^{\mathrm{V} 600 \mathrm{E}}$ mutation (14). Patients with metastatic melanoma restricted to tumors with the B-Raf oncogene have a high rate of tumor response. This was predicted in preclinical models, and the data in humans closely corroborate prior experiences in cell lines and tumor xenograph studies in mice (15-18). Patients without a response to this targeted therapy do not show a decrease in ${ }^{18}$ F-FDG uptake. Therefore, the successful implementation of these targeted therapies in patients with metastatic melanoma is critically dependent on patient stratification and monitoring of treatment course, because only patients with the mutation respond. However, current approaches based on invasive surgical biopsies are not suited for sequential target sampling and analysis. It is infrequent that patients with cancer undergo more than 1 tumor biopsy with any given treatment. Repeated tumor sampling is feasible with fine-needle aspirates, which provide single-cell suspensions amenable to ex vivo analysis using sensitive detection systems. In addition, clinical ${ }^{18}$ F-FDG PET can provide early prediction of treatment response (19). However, PET scans can be performed only every 8-12 wk in routine practice given the limitations of radiation exposure and costs. Advanced microfluid-based technologies sensitive to metabolic changes in small populations of cells obtained from fine-needle aspirates could provide a means to the sequential sampling of tumors from patients.

Compared with imaging systems that rely on the detection of penetrating high-energy photons (20), chargedparticle imaging (e.g., with ${ }^{18} \mathrm{~F}$ positrons) can achieve much higher detection sensitivity and spatial resolution in a compact form factor suitable for radioassays of small cell populations $(21,22)$. Charged-particle imaging systems have typically been dedicated for imaging ex vivo tissue sections, such as in autoradiography (23-25). Less common are systems designed for in vitro applications. One system, developed by the Medipix group, used a silicon pixel array detector for in vitro imaging of ${ }^{14} \mathrm{C}$-L-leucine amino acid uptake in Octopus vulgaris eggs (26). Phosphor imaging plates have also been used to detect charged particles from radiolabeled peptides in microfluidic channels; however, the system required several hours of continuous exposure to produce a single image frame (27). Recent studies have used systems with a charge-coupled device camera to detect light emitted from charged particles interacting with ultrathin phosphors (28) and from Cerenkov radiation (29). The latter work used Cerenkov radiation to image radiolabeled probes inside a microfluidic chip; however, the low sensitivity of the system and the requirement of using a lighttight box make it difficult to perform radioassays in small cell populations.

This paper describes an integrated, miniaturized, in vitro radiometric imaging system, capable of measuring the glucose utilization of a small population (1-200) of cells in a real-time fashion. The radioassay system consists of a microfluidic chip for maintaining and controlling arrays of cells integrated with a $\beta$-camera for real-time imaging of charged particles emitted from radioactive sources in vitro (Fig. 1). The uptake of ${ }^{18} \mathrm{~F}-\mathrm{FDG}$ in melanoma cell lines and primary cells in response to specific drug therapies was monitored in a controlled in vitro microfluidic environment using the $\beta$-camera, with which simultaneous measurements can be obtained from radioactive sources confined within the microfluidic chambers. The advantages of the integrated $\beta$-camera and microfluidic chip are 2 -fold. The system allows for in vitro imaging of cells in a controlled microfluidic platform without major disturbance or removal of the cell cultures - in contrast to conventional radiometric methods that use well-type $\gamma$-counters or liquid scintillation counters. In addition, the integrated system is an exquisitely sensitive technology with low background, providing a significant improvement over conventional well-type $\gamma$-counters (30).

\section{MATERIALS AND METHODS}

\section{$\boldsymbol{\beta}$-Camera}

The $\beta$-camera uses a position-sensitive avalanche photodiode (PSAPD), which provides high sensitivity and spatial resolution in a rugged and compact form factor to detect emitted $\beta$-particles from the microfluidic platform (Supplemental Table 1; supplemental materials are available online only at http://jnm.snmjournals. org). The main advantage of the PSAPD over other $\beta$-imaging technologies is that it uses a simple 4-channel readout to localize $\beta$-particle events, thereby reducing the complexity of the necessary readout electronics. Originally designed for the detection of scintillation light photons (31), the PSAPD has been modified to operate in room light by passivating the top surface with aluminized Mylar (DuPont). The PSAPD was also placed within an inset of an aluminum heating block to heat the $\beta$-camera and regulate the temperature at $37^{\circ} \mathrm{C}$ for in vitro imaging of live cells in the microfluidic platform.

The PSAPD is a silicon semiconductor device (model P1305-P; Radiation Monitoring Devices) (31). It has a $14 \times 14 \mathrm{~mm}$ active area and is made of a monolithic silicon semiconductor, which provides a rugged platform that can withstand repeated use for multiple experiments. The detection layer of the PSAPD, which consists of the neutral drift p-region and depletion region, is approximately $60 \mu \mathrm{m}$ thick. When a charged particle interacts within the silicon p-n junction, charged carriers are produced via ionization and then accelerated by the electric field $\left(2 \times 10^{5}\right.$ $\mathrm{V} / \mathrm{cm}$ ), causing an avalanche effect whereby secondary and tertiary electrons are liberated (Fig. 1A). The avalanche results in a 
A

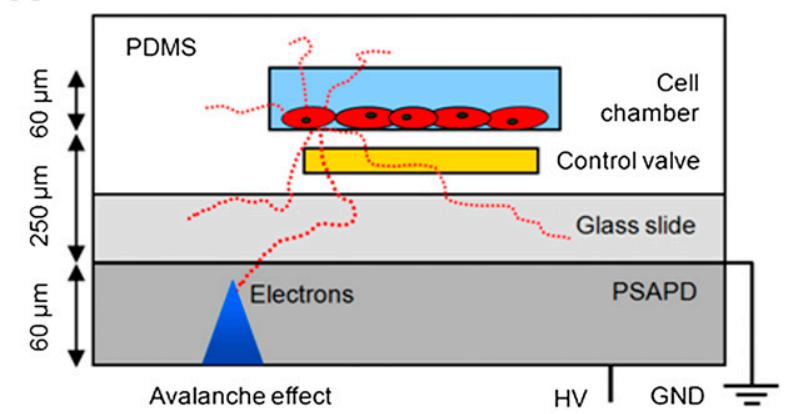

B

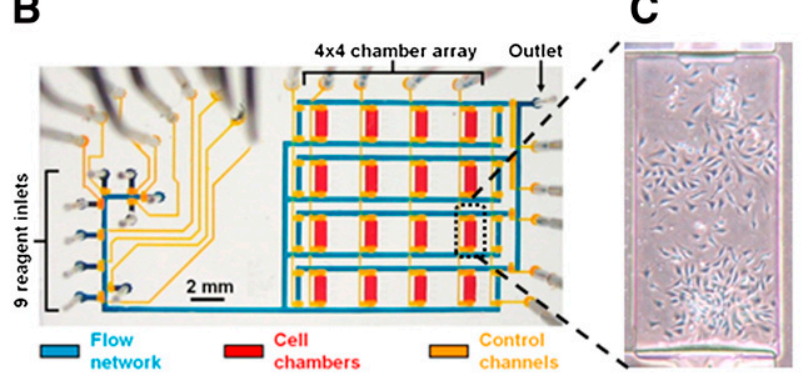

D

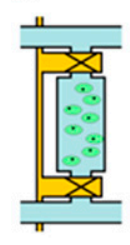

Cell culture on chip

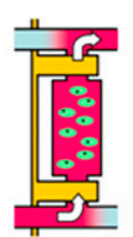

Loading 18F-FDG

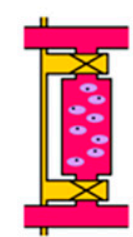

Incubating with ${ }^{18} \mathrm{~F}$ FDG

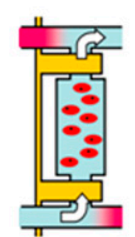

Unloading 18F-FDG

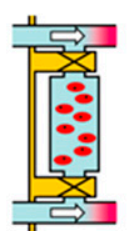

Removing Imaging of ${ }^{18} \mathrm{~F}-\mathrm{FDG}$ trapped ${ }^{18} \mathrm{~F}$. residue

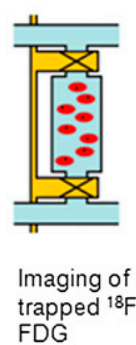

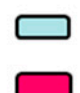

Culture medium

Culture medium with ${ }^{18}$ F-FDG

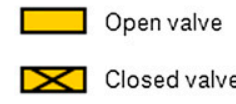

Closed valve

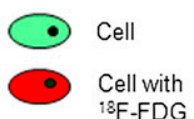

${ }^{18} \mathrm{~F}$-FDG

FIGURE 1. Integrated $\beta$-camera and microfluidic chip for real-time radioassay imaging of glycolysis in small cell populations. (A) Schematic cross-section of $\beta$-camera integrated with microfluidic chip. (B) Micrograph of microfluidic chip loaded with colored dyes. Chamber array $(4 \times 4)$ allows cultured cells to be individually addressable within flow network for parametric study. (C) Micrograph of cells in chamber of microfluidic chip taken with bright-field microscope. (D) Chip operation of an ${ }^{18} \mathrm{~F}-\mathrm{FDG}$ uptake radioassay. From left to right: Cells are allowed to adapt to closed-chamber microenvironment. Valves are opened to allow replacement of culture medium with culture medium containing ${ }^{18} \mathrm{~F}-\mathrm{FDG}$. Cells are incubated in ${ }^{18} \mathrm{~F}-\mathrm{FDG}$ to start ${ }^{18} \mathrm{~F}-\mathrm{FDG}$ uptake. Valves are opened again to wash away extracellular ${ }^{18} \mathrm{~F}-\mathrm{FDG}$ using culture medium. Channel network is washed with culture medium to remove ${ }^{18} \mathrm{~F}$-FDG residing outside cell chamber. ${ }^{18} \mathrm{~F}-\mathrm{FDG}$ uptake of cells is imaged by $\beta$-camera. GND = ground; HV = high voltage; PDMS = polydimethylsiloxane.

signal gain of approximately 1,000-fold and provides a high signal-to-noise ratio to decode the position of ${ }^{18} \mathrm{~F}$ positron events. The position of each charged particle event is localized by taking the weighted average of the 4 corner position signals using a simple algorithm (Supplemental Fig. 1) (32).

\section{Microfluidic Chip}

The microfluidic chip was fabricated from polydimethylsiloxane (33) and placed in contact with the $\beta$-camera platform to directly detect the emitted charged particles. A network of flow channels was interwoven with the microchambers for digital control of samples and reagents with the cell cultures. Nine reagent inlets were needed to supply a variety of biochemical solutions to a particular chamber in an automated fashion through multiple control channels (Figs. 1B-1D). Charged particles are highly attenuated when traversing through materials with densities comparable to water. Therefore, it was necessary to design a microfluidic chip with a minimal substrate thickness separating the radioactive cell cultures from the detector. The microfluidic chip was fabricated using a multilayer soft lithography method and designed with a substrate layer consisting of polydimethylsiloxane (100 $\mu \mathrm{m}$ thick) on top of a glass cover slip (150 $\mu \mathrm{m}$ thick). The overall sensitivity of the $\beta$-camera is highly dependent on the substrate thickness between the source and detector, which will be discussed in a separate publication.

The microfluidic channels and chambers are coated with fibronectin solution to promote cell adhesion onto the polydimethylsiloxane surface, preventing most of the cells from being washed away. When cells adhere to the bottom surface of the cell culture microchamber, they tend to form a thin monolayer where cells may occupy a total volume less than $5 \%$ of the overall microchamber volume. Therefore, to measure the uptake of ${ }^{18} \mathrm{~F}$ FDG into the cell, it was necessary to remove the high background signal due to ${ }^{18} \mathrm{~F}-\mathrm{FDG}$ in the extracellular solution.

\section{Cellular Microfluidic Radioassay}

Image Calibration. As an initial test, the sensitivity of the microfluidic $\beta$-camera was calibrated using a melanoma cancer cell line (M229) incubated in a $4 \times 4$ microchamber array as shown in Figure 1B. Before the microfluidic radioassay, the live cells were loaded into each microchamber with the aid of a brightfield microscope (34). For each radioassay, a mixture of ${ }^{18} \mathrm{~F}-\mathrm{FDG}$ solution was diluted with RPMI 1640 cell culture medium and loaded into the microchambers with a radioactivity concentration of $37 \mathrm{MBq} / \mathrm{mL}(1,000 \mu \mathrm{Ci} / \mathrm{mL})$ and incubated for $30 \mathrm{~min}$. After ${ }^{18} \mathrm{~F}-\mathrm{FDG}$ incubation, cell culture medium was used to wash away the extracellular ${ }^{18} \mathrm{~F}$-FDG from each of the chambers. The efficacy of this washing procedure was measured in a separate experiment, showing that no radioactivity was left in the microfluidic channels after washing. The remaining ${ }^{18} \mathrm{~F}-\mathrm{FDG}$ trapped inside the cells was then imaged using the $\beta$-camera with an acquisition time of $20 \mathrm{~min}$. After the microfluidic radioassay had been completed, a relatively large volume (compared with the microchamber volume) of lysis buffer (10-20 $\mu \mathrm{L})$ was used to lyse the cells from each of the microchambers into plastic vials. After all of the cell cultures were removed from each of the microchambers, the entire chip was imaged for $5 \mathrm{~min}$ with the $\beta$-camera to ensure that no radioactivity remained in the microchambers or microchannels. The total radioactivity in each cell culture sample was then measured for $1 \mathrm{~min}$ using a well-type $\gamma$-counter (1480 Wizard 3; Perkin Elmer), and the counting rate was converted to total radioactivity using a traceable calibration factor according to the National Institute of Standards and Technology for the $\gamma$-counter and branching fraction for ${ }^{18} \mathrm{~F}$. The total radioactivity of each cell culture sample was then correlated with the region of interest (ROI) in the $\beta$-camera image.

Linearity of Microfluidic Radioassay. The effect of extracellular ${ }^{18} \mathrm{~F}-\mathrm{FDG}$ radioactivity concentration on the uptake of ${ }^{18} \mathrm{~F}-\mathrm{FDG}$ 
into cell cultures during the radiotracer incubation period was evaluated. Two melanoma cell lines (M229 and M202) were loaded into each of the chambers with a range of 110-239 cells per chamber. Four different solutions were prepared from the same stock of ${ }^{18} \mathrm{~F}-\mathrm{FDG}$ and diluted using RPMI 1640 cell culture medium to radioactivity concentrations of $0.037,0.370,3.700$, and $37.00 \mathrm{MBq} / \mathrm{mL}(1,10,100$, and $1,000 \mu \mathrm{Ci} / \mathrm{mL}$, respectively). The 4 dilutions were then loaded into the microchambers, and the cells were incubated for $30 \mathrm{~min}$. After ${ }^{18} \mathrm{~F}-\mathrm{FDG}$ incubation, cell culture medium was used to wash away the extracellular ${ }^{18} \mathrm{~F}-\mathrm{FDG}$ from each of the chambers. The remaining ${ }^{18} \mathrm{~F}-\mathrm{FDG}$ trapped inside the cells was then imaged using the $\beta$-camera with an acquisition time of $20 \mathrm{~min}$. From the $\beta$-camera images, ROIs were drawn around the microfluidic chambers, and the total radioactivity per cell was calculated for each chamber.

Single-Cell Microfluidic Radioassay. Two melanoma cell lines (M229 and M202) were loaded into a $4 \times 4$ microchamber array. The 2 left columns of the array were loaded with double-digit numbers of cells, ranging from 12 to 21 cells per chamber. The 2 right columns were loaded with a single-digit number of $0-1$ cell per chamber. The cells were then incubated for $30 \mathrm{~min}$ in a mixture of ${ }^{18} \mathrm{~F}$-FDG solution diluted using RPMI 1640 cell culture medium to a radioactivity concentration of $37 \mathrm{MBq} / \mathrm{mL}(1,000 \mu \mathrm{Ci} / \mathrm{mL})$. Afterward, the same steps were followed as for the linearity evaluation.

\section{Cytotoxic Microfluidic Radioassay}

Melanoma cells M257, M202, M233, and M229 were loaded into the $4 \times 4$ microfluidic chambers, with each cell line placed along a row of chambers. Approximately 150 cells were loaded into each of the microfluidic chambers. One day before the radioassay, the cells were cultured and rested in the microfluidic chambers using RPMI 1640 cell culture medium, with medium replenished every $6 \mathrm{~h}$. PLX4032 stock solution was diluted in RPMI 1640 to $1 \mu \mathrm{M}$, and duplicate samples were treated with the drug (15). The remaining 2 samples from each of the cell lines were used as vehicle controls. After $20 \mathrm{~h}$ of incubation with PLX4032, the microfluidic radioassay was performed. ${ }^{18} \mathrm{~F}-\mathrm{FDG}$ was diluted in a glucose-free RPMI 1640 medium to a radioactivity concentration of $3.7 \mathrm{MBq} / \mathrm{mL}(100 \mu \mathrm{Ci} / \mathrm{mL})$ and loaded into the microfluidic chambers. The ${ }^{18} \mathrm{~F}-\mathrm{FDG}$ solution was loaded into all chambers, and the cells were incubated for $60 \mathrm{~min}$ to ensure adequate uptake. After ${ }^{18}$ F-FDG incubation, cell culture medium was used to wash away the extracellular ${ }^{18} \mathrm{~F}$-FDG from each of the chambers. The remaining ${ }^{18} \mathrm{~F}-\mathrm{FDG}$ trapped inside the cells was then imaged using the $\beta$-camera with an acquisition time of $20 \mathrm{~min}$. The microfluidic radioassay was then repeated for 3 days, and images were acquired with the $\beta$-camera during each day to monitor the response of ${ }^{18} \mathrm{~F}-\mathrm{FDG}$ uptake to PLX4032.

\section{RESULTS}

\section{Image Calibration}

An image of the $\beta$-camera calibration acquisition is shown in Figure 2A, with ROIs drawn around each microfluidic chamber. Because of the variation in the total population of cells in each chamber, ranging from 10 to 40 cells, the total signal in each microchamber also varied proportionally. The average counting rate of each microfluidic chamber measured with the $\beta$-camera was plotted against the total activity within each chamber (Fig. 2B). The absolute sen-

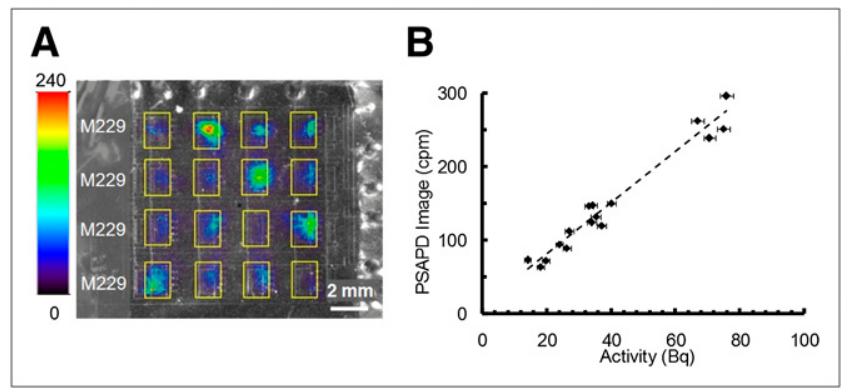

FIGURE 2. Overlay image of ${ }^{18} \mathrm{~F}-\mathrm{FDG}$ uptake in melanoma primary cell line (M229) with $\beta$-camera and optical image of microfluidic chips. All images were evaluated using ROls (yellow rectangles) of equal dimensions. Color bar represents $\beta$-camera image scale and indicates counts per minute (cpm) per square millimeter. (A) ${ }^{18} \mathrm{~F}-\mathrm{FDG}$ uptake image of M229 cell cultures inside microfluidic chambers. (B) Calibration of $\beta$-camera image.

sitivity of the device was $6 \%$ for this particular microfluidic chip geometry using a linear fit of the data. The calibration factor was then used to convert the $\beta$-camera counting rates to total radioactivity for all imaging experiments performed with this microfluidic chip design.

\section{Linearity of Microfluidic Radioassay}

The $\beta$-camera image of ${ }^{18} \mathrm{~F}$-FDG uptake for cell cultures incubated in varying levels of radioactivity concentration is shown in Figure 3A. Because of the limitations of the display, the full dynamic range of the $\beta$-camera cannot be
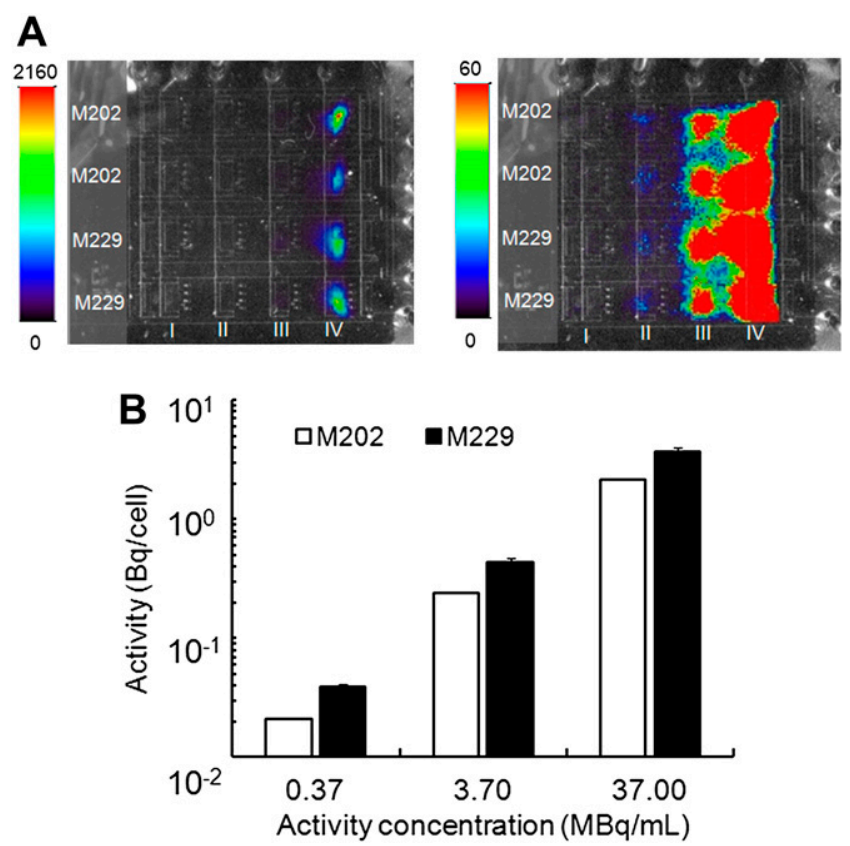

FIGURE 3. (A) Same $\beta$-camera image displayed with 2 different color-intensity scales. In each $\beta$-camera image, ${ }^{18} \mathrm{~F}$-FDG uptake was measured for cell cultures incubated with ${ }^{18} \mathrm{~F}-\mathrm{FDG}$ radioactivity concentrations of $0.037,0.370,3.700$, and $37.00 \mathrm{MBq} / \mathrm{mL}$ (I, II, III, and IV, respectively). (B) ${ }^{18} \mathrm{~F}-\mathrm{FDG}$ uptake values are given as mean ( \pm SEM) activity normalized to number of cells in each chamber. 
shown in a single image. The 2 images shown in Figure $3 \mathrm{~A}$ are of the same data, with different maximum-color-intensity scales. For both cell lines (M202 and M229), the culture samples incubated with $0.037 \mathrm{MBq} / \mathrm{mL}(1 \mu \mathrm{Ci} / \mathrm{mL})$ had ${ }^{18}$ F-FDG uptake below the detection limit of the system. For the culture samples incubated in the 3 higher radioactivity concentrations, a linear correlation between the ${ }^{18} \mathrm{~F}-\mathrm{FDG}$ radioactivity concentration and the amount of ${ }^{18} \mathrm{~F}$-FDG uptake per cell for both cell lines (Fig. 3B) was observed after normalizing for the number of cells per microchamber. The uptake measured for M229 cells was $0.04 \pm 0.00,0.43 \pm 0.04$, and $3.70 \pm 0.27 \mathrm{~Bq} /$ cell for each of the 3 highest radioactivity concentrations $(0.370,3.700$, and $37.00 \mathrm{MBq} / \mathrm{mL})$, respectively. For M202 cells, the average uptake values were $0.02 \pm 0.00$, $0.24 \pm 0.00$, and $2.13 \pm 0.04 \mathrm{~Bq} /$ cell, respectively, for each of the 3 highest radioactivity concentrations. All error values are reported as SEM.

\section{Single-Cell Microfluidic Radioassay}

A $\beta$-camera image of the ${ }^{18} \mathrm{~F}$-FDG uptake in single-cell cultures is shown in the two right columns of the microfluidic chip in Figure 4A. Again, because of the limitations of the display, the full dynamic range of the $\beta$-camera cannot be shown in a single image. The 2 images shown in Figure 4A are of the same data, with different maximumcolor-intensity scales. For microfluidic chambers populated by a single cell, the ${ }^{18} \mathrm{~F}-\mathrm{FDG}$ uptake was $2.85 \pm 0.23$ and $2.22 \pm 0.49 \mathrm{~Bq} /$ cell for M229 and M202 cell lines, respectively (Fig. 4B). Three of the microfluidic chambers contained no cells and hence had no signal. The microfluidic chambers with a population of 10 cells or greater had ${ }^{18} \mathrm{~F}$ FDG uptake of $3.15 \pm 0.10$ and $2.14 \pm 0.25 \mathrm{~Bq} /$ cell for M229 and M202 cell lines, respectively.

\section{Cytotoxic Microfluidic Radioassay}

The total number of cells in each culture was counted, and proliferation rates over the course of the experiment were consistent for each of the cell lines treated with drug. The B-Raf ${ }^{\mathrm{V} 600 \mathrm{E}}$ mutant melanoma cell line M229 cultured in PLX4032 showed a decrease in proliferation rates, compared with the vehicle control cell cultures that were not treated with PLX4032, whereas the M233, M257, and M202 cell lines showed little or no response to PLX4032 exposure (Supplemental Fig. 2), as previously described using macroscopic assays (15). A qualitative decrease in the ${ }^{18}$ F-FDG uptake signal for M229 cells treated with $1 \mu \mathrm{M}$ PLX4032, compared with vehicle control, can be seen in Figure 5B. ROIs were then drawn around the microfluidic chambers, and the total radioactivity per cell was calculated for each chamber. The highly sensitive M229 cells treated with $1 \mu \mathrm{M}$ of PLX4032, compared with vehicle controls, showed a $30.0 \% \pm 3.2 \%$ decrease in ${ }^{18}$ F-FDG uptake per cell on day 1, as shown in Figure 5C. Repeated experiments on the same M229 cell cultures, compared with vehicle controls, showed that additional drug treatments on days
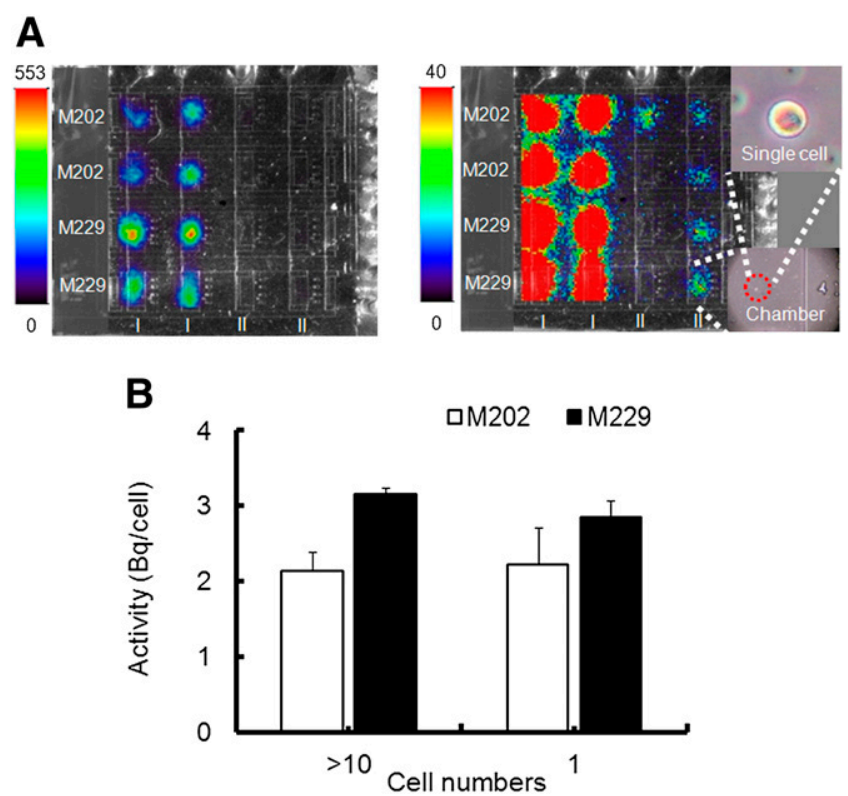

FIGURE 4. (A) Same $\beta$-camera image displayed with 2 different color-intensity scales. In each image, the 2 leftmost columns (I) show ${ }^{18} \mathrm{~F}-\mathrm{FDG}$ uptake in chambers with double-digit numbers of cells. The 2 rightmost columns (II) show image of ${ }^{18} \mathrm{~F}-\mathrm{FDG}$ uptake in chambers with single cell. Micrograph of selected chamber and zoom-in section showing same chamber that has only single cell. (B) ${ }^{18} \mathrm{~F}-\mathrm{FDG}$ uptake values are given as mean ( \pm SEM) activity normalized to number of cells in each chamber.

2 and 3 also decreased the ${ }^{18} \mathrm{~F}-\mathrm{FDG}$ uptake per cell $(31.4 \% \pm 5.1 \%$ and $29.9 \% \pm 9.3 \%)$. As expected, there was no decrease in ${ }^{18} \mathrm{~F}$-FDG uptake per cell in the other 3 melanoma cell lines when treated with drug, as correlates with their lack of response with exposure to the B-Raf inhibitor PLX4032.

\section{DISCUSSION}

A combination of the sensitivity of the $\beta$-camera and the precision with which the microfluidic system controls the microenvironment allows for radioassays of a single-cell culture. ${ }^{18}$ F-FDG uptake per cell for both M229 and M202 melanoma cell lines was consistent for cell populations ranging from 200 cells down to a single cell when incubated with a radioactivity concentration of $37 \mathrm{MBq} / \mathrm{mL}$ (1,000 $\mu \mathrm{Ci} / \mathrm{mL})$ during the radiotracer incubation period.

Melanomas can have 1 of 3 driver oncogenic events in the mitogen-activated protein kinase pathway: kit mutations (3\%), N-Ras mutations (15\%), and B-Raf mutations $(60 \%)$ $(12,35)$. These are mutually exclusive mutations, suggesting a dominant oncogenic event in the development of this cancer and a likely therapeutic target (12). We took advantage of the specific antitumor effects of a novel B-Raf inhibitor, PLX4032, in melanoma cell lines with defined oncogenic mutations as a way to test whether the $\beta$-camera and microfluidic chip could be used to assess differential therapeutic activity (15). M229 has a homozygous BRaf $^{\mathrm{V} 600 \mathrm{E}}$ mutation and is highly sensitive to PLX4032, with 
A

FIGURE 5. ${ }^{18} \mathrm{~F}-\mathrm{FDG}$ radioassay of melanoma cell lines treated with B-Raf inhibitor drug. (A) Chip operation for ${ }^{18} \mathrm{~F}-\mathrm{FDG}$ uptake radioassay of drug-treated melanoma cells. (Left to right) Cells are allowed to adapt to closed-chamber microenvironment. Valves are opened to allow replacement of culture medium by culture medium containing PLX4032. Cells are incubated in ${ }^{18} \mathrm{~F}-\mathrm{FDG}$ to start ${ }^{18} \mathrm{~F}-\mathrm{FDG}$ uptake. Valves are opened again to wash away extracellular ${ }^{18} \mathrm{~F}-\mathrm{FDG}$ using culture medium. Channel network is washed with culture medium to remove ${ }^{18} \mathrm{~F}-\mathrm{FDG}$ residing outside cell chamber. ${ }^{18} \mathrm{~F}-\mathrm{FDG}$ uptake of cells is imaged by $\beta$-camera. (B) Overlay image of ${ }^{18} \mathrm{~F}-\mathrm{FDG}$ uptake with $\beta$-camera and optical image of microfluidic chip. (C) ${ }^{18} \mathrm{~F}-\mathrm{FDG}$ uptake values are given as ratio of mean ( \pm SEM) activity normalized to number of cells in each chamber between drug-treated cells and vehicle control.

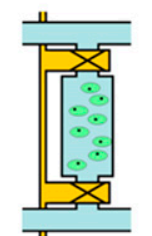

Cell culture on chip

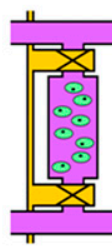

Drug

PLX4032 incubation

Culture medium with PLX4032

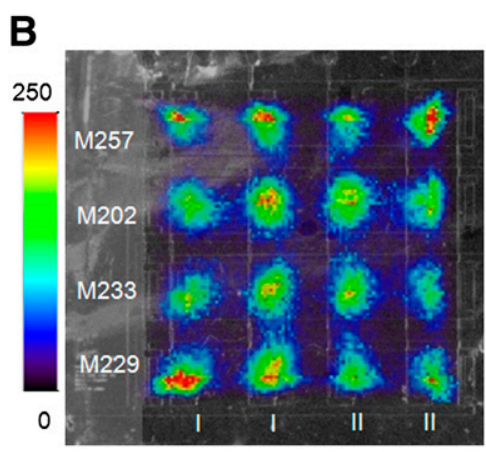

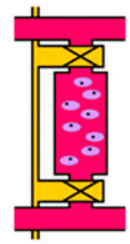

Incubating with ${ }^{18} \mathrm{~F}$ FDG

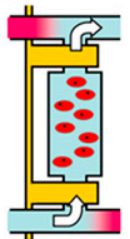

Unloading 18F-FDG

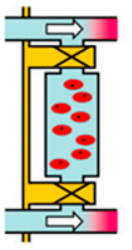

Removing ${ }^{18 F-F D G}$ residue

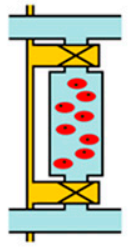

Imaging of trapped ${ }^{18} \mathrm{~F}$ FDG a 50\% inhibition concentration of $0.2 \mu \mathrm{M}$, whereas M233 has a heterozygous B-Raf ${ }^{\mathrm{V} 600 \mathrm{E}}$ mutation but is resistant to this therapy, with a $50 \%$ inhibition concentration of more than $10 \mu \mathrm{M}$ (Supplemental Fig. 3A). M202 has a mutually exclusive N-Ras Q61 L mutation, and M257 is wild-type for both B-Raf and N-Ras, with both cell lines also being resistant to PLX4032 (15).

Macroscopic radioassays were also performed as a way to compare and validate the microfluidic results showing a decrease in ${ }^{18} \mathrm{~F}-\mathrm{FDG}$ uptake of M229 cells treated with 1 $\mu M$ PLX4032 (Supplemental Fig. 3B). There are several differences in protocol between the microfluidic and macroscale approaches. Compared with the macroscopic wellplate experiments, in each microfluidic chamber, a smaller population of cells was cultured. Therefore, a higher radioactivity concentration was used with the $\beta$-camera experiments, to increase the total signal available from each sample. In addition, the limited volume of each microfluidic chamber also necessitated that cell medium be replenished every $6 \mathrm{~h}$ during the microfluidic radioassay. These differences in protocol may affect the condition of the cells and the uptake of ${ }^{18} \mathrm{~F}-\mathrm{FDG}$ radiotracer when comparing the results of the macroscale and microfluidic radioassays.

An advantage of the microfluidic platform is that it can provide a method for maintaining cell cultures for long periods (days) in an environment in which perturbations can be precisely controlled (36). In contrast, macroscopic studies can perform only a single radioassay on a given cell culture sample because each measurement is an endpoint study requiring that the cells be disturbed or removed from the culture environment. Compared with conventional mac- roscopic radioassays, which offer high sensitivity for radioactive detection using large samples, the $\beta$-camera and microfluidic chip provide digital control of small populations of cell cultures and the ability to perform radioassays of live cells in real time and in vitro.

\section{CONCLUSION}

We have developed a $\beta$-camera for imaging charged particles emitted from radiotracers in vitro using a solid-state PSAPD detector integrated with a microfluidic chip that can provide a platform for imaging live cell cultures. The high sensitivity of the $\beta$-camera allows for radioassays of small cell populations down to a single-cell level. The system provides researchers with a new tool to radioassay small cell populations that can complement traditional in vitro radioassays for screening diagnostic and therapeutic radiopharmaceuticals.

A key issue in early clinical development of novel specific pathway inhibitors is how the drug modulates its target and delivers the desired pharmacodynamic effects (14). In solid tumors, derivation of pharmacodynamic information is limiting, and most drugs are developed in a masked way (without evidence of the dose level at which the drug modulates its target). Tumor biopsies and molecular imaging using PET have been the best approaches to providing pharmacodynamic information. However, because of the patient inconvenience, risks of complications with repeated surgical or core biopsies, and the limitations of high radiation exposure and costs with repeated PET scans, these approaches are not easily used in routinely repeated procedures. 
The integrated $\beta$-camera and microfluidic chip provide an assay platform that could challenge the traditional drug development paradigm by providing a means to quickly and repeatedly define in vitro effects of the kinase inhibitor on its target, starting from small tumor samples obtained by fine-needle aspiration - a procedure that is amenable to repeated tumor sampling.

\section{DISCLOSURE STATEMENT}

The costs of publication of this article were defrayed in part by the payment of page charges. Therefore, and solely to indicate this fact, this article is hereby marked "advertisement" in accordance with 18 USC section 1734.

\section{ACKNOWLEDGMENTS}

This work was funded in part by a National Cancer Institute grant (U54 CA119347), the Caltech-UCLA Joint Center for Translational Medicine, a contract from the Office of Biological and Environmental Research of the Department of Energy (DE-FC03-02ER63420), and a National Institutes of Health Biomedical Imaging and Bioengineering training grant (5T32EB002101). Nam T. Vu and Arion F. Chatziioannou are owners of Sofie Biosciences, Inc., which has licensed the patent covering the integrated microfluidic $\beta$-camera technology from the University of California. Nam $\mathrm{T}$. Vu is currently employed by Sofie Biosciences, Inc.

\section{REFERENCES}

1. Phelps ME. Positron emission tomography provides molecular imaging of biological processes. Proc Natl Acad Sci USA. 2000;97:9226-9233.

2. Avril N, Menzel M, Dose J, et al. Glucose metabolism of breast cancer assessed by ${ }^{18} \mathrm{~F}$-FDG PET: histologic and immunohistochemical tissue analysis. $\mathrm{J} \mathrm{Nucl}$ Med. 2001;42:9-16.

3. Torizuka T, Tamaki N, Inokuma T, et al. In vivo assessment of glucose metabolism in hepatocellular carcinoma with FDG-PET. J Nucl Med. 1995;36:1811-1817.

4. Warburg O. On the origin of cancer cells. Science. 1956;123:309-314.

5. Gatenby RA, Gillies RJ. Why do cancers have high aerobic glycolysis?. Nat Rev Cancer. 2004;4:891-899.

6. Cherry SR, Sorenson JA, Phelps ME. Physics in Nuclear Medicine. Maryland Heights, MO: Elsevier Science; 2003.

7. Sun J, Masterman-Smith M, Graham NA, et al. A microfluidic platform for systems pathology: multiparameter single-cell signaling measurements of clinical brain tumor specimens. Cancer Res. 2010;70:6128-6138.

8. Huang B, Wu H, Bhaya D, et al. Counting low-copy number proteins in a single cell. Science. 2007;315:81-84.

9. Wheeler AR, Throndset WR, Whelan RJ, et al. Microfluidic device for singlecell analysis. Anal Chem. 2003;75:3581-3586.

10. Vander Heiden MG, Cantley LC, Thompson CB. Understanding the Warburg Effect: the metabolic requirements of cell proliferation. Science. 2009;324:1029-1033.

11. Yun J, Rago C, Cheong I, et al. Glucose deprivation contributes to the development of KRAS pathway mutations in tumor cells. Science. 2009;325:1555-1559.

12. Gray-Schopfer V, Wellbrock C, Marais R. Melanoma biology and new targeted therapy. Nature. 2007;445:851-857.

13. Davies H, Bignell GR, Cox C, et al. Mutations of the BRAF gene in human cancer. Nature. 2002;417:949-954.
14. Flaherty K, Puzanov I, Kim K, et al. Inhibition of mutated, activated BRAF in metastatic melanoma. N Engl J Med. 2010;363:809-819.

15. Sondergaard JN, Nazarian R, Wang Q, et al. Differential sensitivity of melanoma cell lines with BRAF V600E mutation to the specific B-Raf inhibitor PLX403. J Transl Med. 2010;8:39.

16. Bollag G, Hirth P, Tsai J, et al. Clinical efficacy of a B-RAF inhibitor requires substantial ERK pathway inhibition in BRAF-mutant melanoma. Nature. 2010; 467:596-599.

17. Joseph EW, Pratilas CA, Poulikakos PI, et al. The RAF inhibitor PLX4032 inhibits ERK signaling and tumor cell proliferation in a V600E BRAF-selective manner. Proc Natl Acad Sci USA. 2010;107:14903-14908.

18. Tsai J, Lee JT, Wang W, et al. Discovery of a selective inhibitor of oncogenic B-Raf kinase with potent antimelanoma activity. Proc Natl Acad Sci USA. 2008; 105:3041-3046.

19. Weber WA, Grosu AL, Czernin J. Technology insight: advances in molecular imaging and an appraisal of PET/CT scanning. Nat Clin Pract Oncol. 2008;5: $160-170$.

20. Fischer BM, Olsen MWB, Ley CD, Klausen TL, Mortensen J, Højgaard L. How few cancer cells can be detected by positron emission tomography? A frequent question addressed by an in vitro study. Eur J Nucl Med Mol Imaging. 2006; 33:697-702.

21. Vu NT, Yu ZTF, Silverman RW, et al. Performance of an integrated microfluidic chip and position sensitive APD for the detection of beta emitting probes in cell cultures. 2007 IEEE Nucl Sci Symp Conf Rec. 2007:4057-4061.

22. Vu NT, Chung YH, Yu ZTF, et al. Direct detection of beta particles on a microfluidic chip using position sensitive APDs. 2006 IEEE Nucl Sci Symp Conf Rec. 2006:3536-3539.

23. Cabello J, Bailey A, Kitchen I, et al. Digital autoradiography using room temperature CCD and CMOS imaging technology. Phys Med Biol. 2007;52:49935011.

24. Mettivier G, Montesi MC, Russo P. First images of a digital autoradiography system based on a Medipix2 hybrid silicon pixel detector. Phys Med Biol. 2003;48:N173-N181.

25. Russo P, Lauria A, Mettivier G, et al. ${ }^{18}$ F-FDG positron autoradiography with a particle counting silicon pixel detector. Phys Med Biol. 2008;53:6227-6243.

26. Bertolucci E, Conti M, Mettivier G, Montesi MC, Russo P. BETAview: a digital $\beta$-imaging system for dynamic studies of biological phenomena. Nucl Instr Meth Phys Res A. 2002;478:109-113.

27. Lavén M, Velikyan I, Djodjic M, et al. Imaging of peptide adsorption to microfluidic channels in a plastic compact disc using a positron emitting radionuclide. Lab Chip. 2005;5:756-763.

28. Chen L, Gobar LS, Knowles NG, Liu Z, Gmitro AF, Barrett H. Direct imaging of radionuclide-produced electrons and positrons with an ultrathin phosphor. $\mathrm{J} \mathrm{Nucl}$ Med. 2008;49:1141-1145.

29. Cho JS, Taschereau R, Olma S, et al. Cerenkov radiation imaging as a method for quantitative measurements of beta particles in a microfluidic chip. Phys Med Biol. 2009;54:6757-6771.

30. Fang C, Wang Y, Vu NT, et al. Integrated microfluidic and imaging platform for a kinase activity radioassay to analyze minute patient cancer samples. Cancer Res. 2010;70:8299-8308.

31. Shah KS, Farrell R, Grazioso R, Harmon ESA-H ES., Karplus EA-K, E. Positionsensitive avalanche photodiodes for gamma-ray imaging. IEEE Trans Nucl Sci. 2002;49:1687-1692.

32. Zhang J, Olcott PD, Levin CS. A new positioning algorithm for position-sensitive avalanche photodiodes. IEEE TransNucl Sci. 2007;54:433-437.

33. Unger MA, Chou H-P, Thorsen T, Scherer A, Quake SR. Monolithic microfabricated valves and pumps by multilayer soft lithography. Science. 2000;288: $113-116$.

34. Kamei K, Guo S, Yu Z, et al. An integrated microfluidic culture device for quantitative analysis of human embryonic stem cells. Lab Chip. 2009;9:555563.

35. Curtin JA, Fridlyand J, Kageshita T, et al. Distinct sets of genetic alterations in melanoma. N Engl J Med. 2005;353:2135-2147.

36. Yu ZT, Kamei K, Takahashi H, et al. Integrated microfluidic devices for combinatorial cell-based assays. Biomed Microdevices. 2009;11:547-555. 